\title{
Imaging of Sodium MRI for Therapy Evaluation of Brain Metastase with Cyberknife at 7T: A Case Report
}

Lichao Huang ${ }^{1}$, Zihao Zhang ${ }^{2}$, Baolin $\mathrm{Qu}^{3}$, Zhiqiang Cui ${ }^{1}$, Yao Wang ${ }^{3}$, Jiwei $\mathrm{Li}^{3}$, Jinyuan Wang ${ }^{3}$, Zhentao Zuo ${ }^{4}$, Yan Zhuo ${ }^{4}$, Xinguang Yu ${ }^{1}$, Zhipei Lin ${ }^{1}$, Longsheng Pan ${ }^{1}$

1. Department of Neurosurgery, PLA General Hospital, Beijing, CHN 2. State Key Laboratory of Brain and Cognitive Science, Institute of Biophysics, Chinese Academy of Sciences 3. Department of Radiation Oncology, PLA General Hospital, Beijing, CHN 4. Institute of Biophysics, State Key Laboratory of Brain and Cognitive Science Chinese Academy of Sciences

Corresponding author: Longsheng Pan, panls301@163.com

\begin{abstract}
Herein we describe the case of an elderly patient who presented with a recent history of impaired vision of the right eye around three months due to brain lesions. He was diagnosed with liver cancer and underwent surgery three months prior. The pathological result is hepatocellular carcinoma. Magnetic resonance imaging (MRI) revealed the diagnosis of brain to be metastatic. The patient selected CyberKnife (Accuray Incorporated, Sunnyvale, USA) radiosurgery for the brain lesion since his physical conditions are not suitable for craniotomy. We adapt the imaging of sodium MRI and proton diffusion mapping at 7T MR system to evaluate the efficacy following CyberKnife early stage treatment. To date, we find the tissue sodium concentration (TSC) changes with the time whereas the proton MRI has no significant change within one month. The time course of sodium concentration in the tumor showed a dramatic increase in the treated brain tumor compared to the pretreatment sodium concentration and 48 hours after stereotactic

radiosurgery (SRS), which is correlated to the period of the radiotherapy-induced cellular necrosis. This case demonstrates the possibility of sodium MRI as a biomarker for monitoring early radiotherapy for assessing tumor cellularity.
\end{abstract}

Received 03/06/2018

Review began 03/12/2018 Review ended 04/08/2018 Published 04/18/2018

๑ Copyright 2018

Huang et al. This is an open access article distributed under the terms of the Creative Commons Attribution License CC-BY 3.0., which permits unrestricted use, distribution, and reproduction in any medium, provided the original author and source are credited.
Categories: Radiation Oncology, Neurosurgery

Keywords: sodium mri, brain metastase, cyberknife, tissue sodium concentration (tsc)

\section{Introduction}

Stereotactic radiosurgery (SRS) is an established and effective treatment for brain metastases (BM). The proton MRI is used to evaluate the volume of tumor change after radiotherapy by RECIST (Response Evaluation Citeria in Solid Tumors). However, the changes in the level of tumor cells could not be reflected. In contrast, sodium MRI could reveal tumor microenvironment. Schepkin et al. have investigated the changes of sodium signal of rat brain tumor model in response to the chemotherapy [1]. In our study, we investigated the sequence of changes in the sodium MRI signal in the brain metastasis following CyberKnife radiosurgery and the main focus is on the evaluation for the early response after SRS procedure with CyberKnife.

\section{Case Presentation}

A 60-year-old man who presented with a recent history of impaired vision of right eye for about 3 months due to brain lesions. He was diagnosed with liver cancer and underwent surgery three months ago. The pathological result is hepatocellular carcinoma. His right eye showed impaired vision and the proton MRI revealed brain metastasis located in the right orbital part (Figure 1).

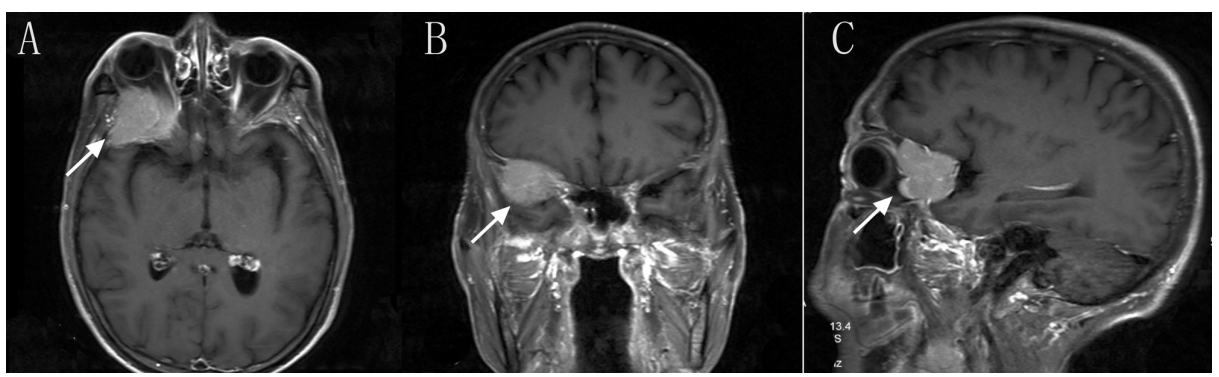

FIGURE 1: Initial T1 Contrast-enhanced magnetic resonance imaging

Axial (A),Coronal (B) and Sagittal (C) T1 Contrast-enhanced MRI imaging revealed an cranio-orbital 


\section{Cureus}

tumor(arrow)

The patient was not suitable for the surgical excision due to his poor physical conditions after liver tumor resection. CyberKnife radiosurgery was delivered at a dose of 22.5 Gy in three fractions (Figure 2-3).

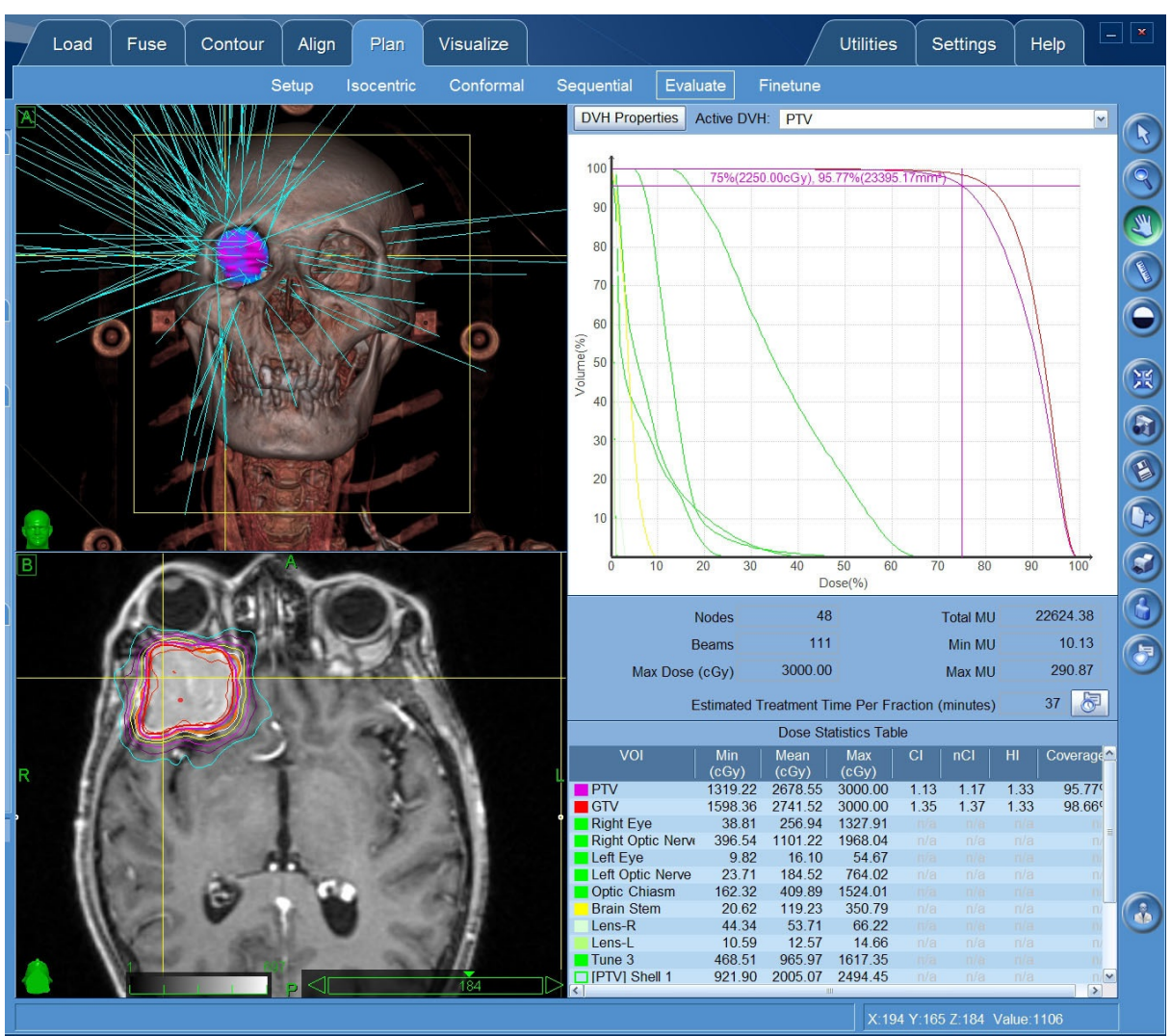

FIGURE 2: CyberKnife radiosurgery planing 


\section{Cureus}

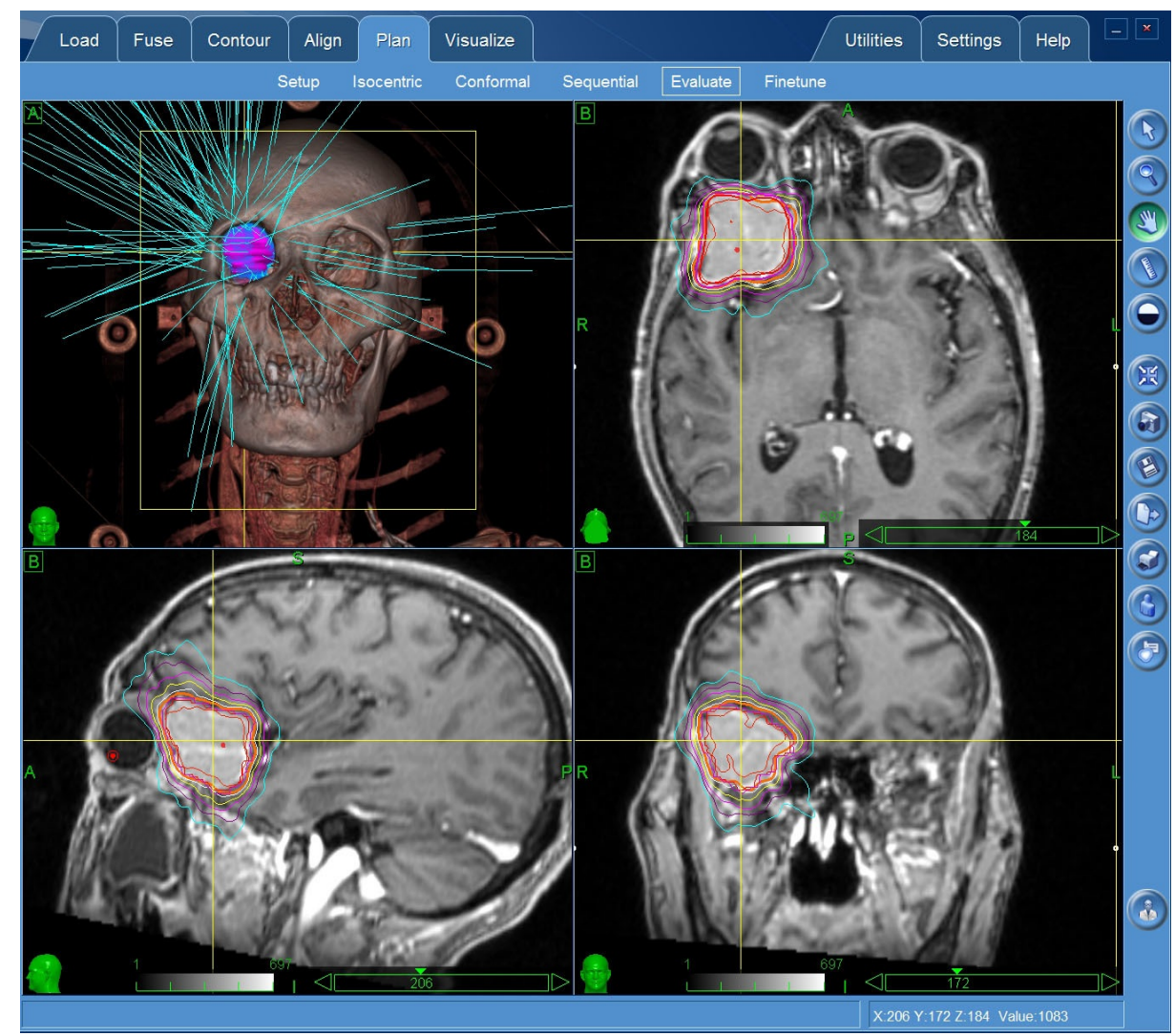

FIGURE 3: Cyberknife radiosurgery planing

Both sodium and proton MRI (Siemens Magnetom 7T, Erlangen, Germany) were performed periodically at $7 \mathrm{~T}$ by the time schedule of pre-treatment, 48 hours after SRS, with one week follow up and one month follow up. The scan parameters are listed in Table 1.

\begin{tabular}{|llll|}
\hline \multicolumn{2}{|c|}{ Scan Parameters } & & \\
\hline Sequence & FOV $(\mathrm{mm})$ & Resolution $(\mathrm{mm})$ & Time $(\mathrm{min})$ \\
T1 & $224 \times 203 \times 179$ & $0.70 \times 0.70 \times 0.70$ & $5: 06$ \\
T2 & $190 \times 199 \times 103$ & $0.26 \times 0.26 \times 2.5$ & $7: 28$ \\
FIAIR & $220 \times 199 \times 117$ & $0.43 \times 0.43 \times 3.0$ & $7: 58$ \\
DWI & $196 \times 196 \times 120$ & $2.0 \times 2.0 \times 2.0$ & $4: 48$ \\
Sodium & $224 \times 224 \times 224$ & $3.5 \times 3.5 \times 3.5$ & $\sim 14: 00$ \\
\hline
\end{tabular}

\section{TABLE 1: Scan parameters}

FOV (Field of view)

FLAIR (Fluid Attenuated Inversion Recovery)

DWI (Diffusion Weighted Imaging)

The sodium signal in the tumor is obviously higher than the opposite normal brain tissue within one month after SRS (E-H). However, the T2 magnetic resonance imaging reveals no obvious changes (A-D) (Figure 4). 


\section{Cureus}
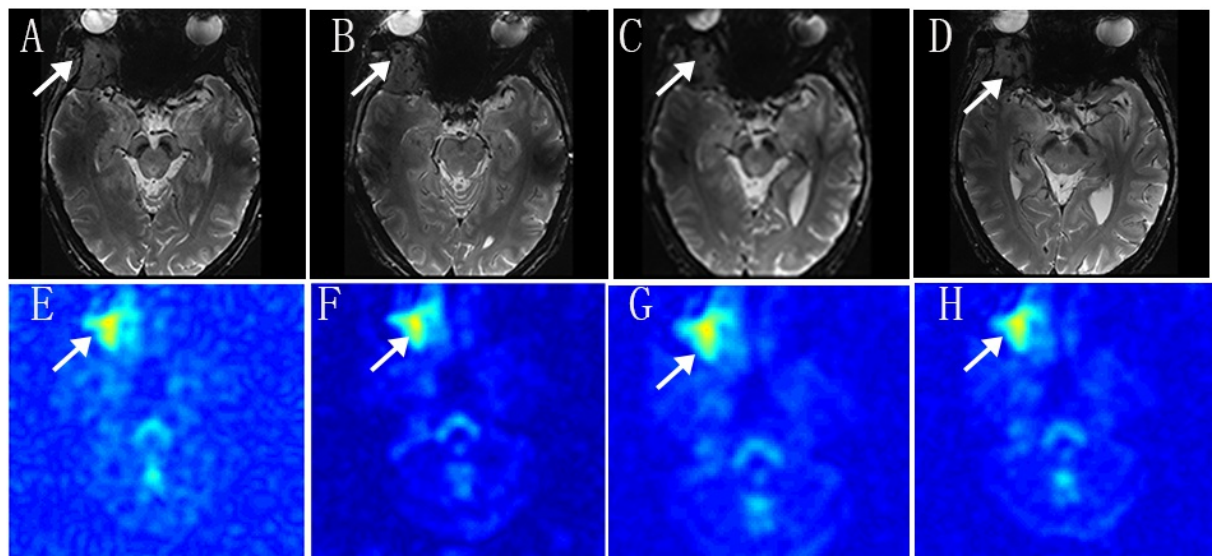

FIGURE 4: T2-weight imaging and sodium MRI.

Axial T2-weight MRI with pretreatment (A), 48 hours after treatment (B), post-treatment of 1 week (C) and post-treatment of 1 month (D). Axial Sodium MRI Images with pretreatment (E) 48 hours after treatment $(B)$, post-treatment of 1 week (C), post-treatment of 1 month (D)

The quantified sodium signal intensity in MRI was shown in Table 2.

\begin{tabular}{|c|c|c|c|c|}
\hline Scan & untreated & 48 hours & one week & one month \\
\hline Tumor & 1599.729244 & 1619.115858 & 1551.354553 & 1703.269578 \\
\hline Ventricle & 1195.083046 & 1188.588231 & 1226.508085 & 1233.800309 \\
\hline Ratio & 1.338592535 & 1.362217643 & 1.26485473 & 1.380506688 \\
\hline
\end{tabular}

TABLE 2: Sodium MRI Signal Intensity

The time course of sodium signal intensity in the tumor showed a dramatic increase in the treated brain tumor compared to the pretreatment and SRS within 48 hours. And the signal intensity decreased at one week after SRS compared to 48 hours after SRS. However, the increased signal intensity was observed at one month. The TSC ratio of tumor to cerebrospinal fluid (CSF) is much more intuitive (Figure 5).
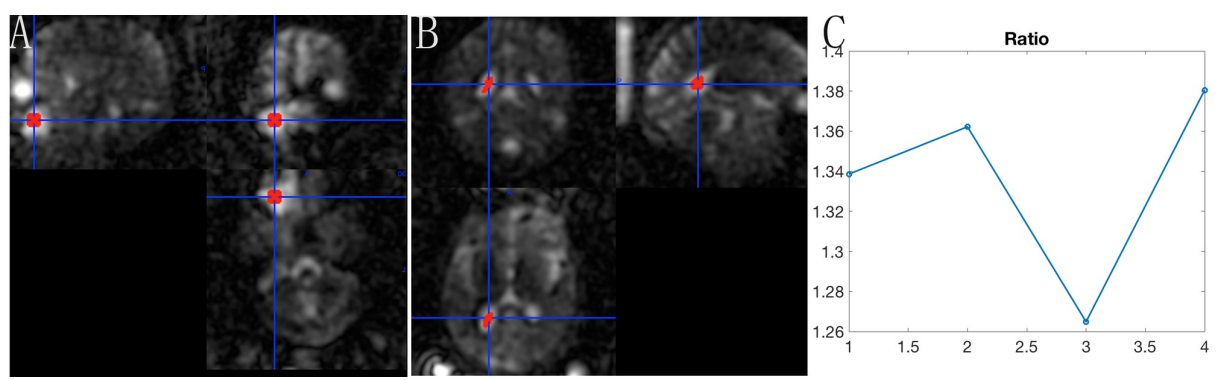

\section{FIGURE 5: The TSC ratio of tumor and cerebrospinal fluid}

The sodium signal intensity of ROI (region of interest) located in the center of tumor (A) as well as the sodium signal intensity of CSF in lateral ventricle (B) were quantified. The ratio of sodium signal intensity in tumor and CSF is shown clearly (C).

\section{Discussion}

Stereotactic radiosurgery (SRS) is becoming a recognized, fashionable treatment option for brain metastases with reported excellent local control rates. And the multi-session SRS delivered by CyberKnife is an effective option for the treatment of intraorbital lesions with tumor size shrinkage, pain relieving, and vision preserved. The fractionated scheme applied in our study with a dose of $22.5 \mathrm{~Gy}$ in three fractions is based on the indication of SRS. 
The therapeutic responses of brain tumors are conventionally assessed by size and contrast enhancement characteristics on CT or MRI images. With the advancement of molecular imaging, biomarkers are adopted with its ability to reflect the early prediction of treatment outcome since cellular changes occur earlier, before tumor shrinkage observed [2]. Sodium ions $(23 \mathrm{Na}+)$ are vital components in human brains, and the cellular homeostasis process is through coupled exchange with potassium ions $\mathrm{K}+$ between the intra- and extra-cellular compartments by $\mathrm{Na}+\mathrm{K}+$-ATPase (sodium-potassium pump) [3]. Dysregulation of the sodium-potassium pump, or of ATP-dependent processes in the cells will provoke dysregulation of ion homeostasis and therefore leads to an increase of intracellular sodium concentration (TSC) as the gradient cannot be sustained anymore, and furthermore to cell death [4]. These bio scales (TSC) can monitor the spatial distribution of tissue responses to radiation treatment on at least a weekly basis and could be used to guide adaptive radiation treatment for each patient and avoid excessive radiation when no response can be achieved [5]. Our study was to investigate the TSC change before the tumor shrinkage in humans.

Data presented in this our study reveal that the sodium signal in pretreatment tumor is obviously higher than the opposite normal brain tissue. Because the cell membrane depolarization precedes cell division in proliferative neoplastic tissue, leads to an increase in the intracellular sodium concentration and a concomitant rise in the total sodium concentration in the tumor tissue [6]. The first TSC peaks after CyberKnife radiosurgery within 48 hours. Sodium overload may have a strong connection with apoptosis and even initiate apoptosis itself [7]. We consider that the first TSC peak in 48 hours is related to radiotherapyinduced apoptosis. The second TSC peak in one month may be related to tumor recurrence, however, further pathological results could not be obtained. Further MRI examination will be performed by image findings.

The sodium MRI offers complementary information which could be quantitatively measured and it is noninvasive. Even though the clinical value of sodium MRI as a complement to proton MRI or other imaging modalities such as PET and CT is still under investigation [8], our study shows the clinical value with due to the biochemical information provided.

\section{Conclusions}

In conclusion, we present a case of complicated brain metastatic treated with CyberKnife radiosurgery. The case demonstrates the possibility of sodium MRI as a biomarker for monitoring early radiotherapy for assessing tumor cellularity. The noninvasive and rapid feedback may be used to guide the management of brain tumors.

\section{Additional Information \\ Disclosures}

Human subjects: Consent was obtained by all participants in this study. The Institutional Review Board of Beijing MRI Center for Brain Research issued approval 2004-IRB-001. I confirm that the purpose of the research, the study procedures and the possible risks and discomforts as well as potential benefits that I may experience have been explained to me. All my questions have been satisfactorily answered. I have read this consent form. My signature below indicates my willingness to participate in this study. Conflicts of interest: In compliance with the ICMJE uniform disclosure form, all authors declare the following: Payment/services info: All authors have declared that no financial support was received from any organization for the submitted work. Financial relationships: All authors have declared that they have no financial relationships at present or within the previous three years with any organizations that might have an interest in the submitted work. Other relationships: We want to know if the co-author Zihao Zhang could be listed as co-first author?.

\section{Acknowledgements}

The authors thank Fernando E. Boada from New York University for providing the twisted projection imaging sequence and tunning the scanning parameters. This work was supported in part by the Ministry of Science and Technology of China grant (2015CB351701), and the National Nature Science Foundation of China grant (91132302). In the meanwhile, the authors acknowledge Dr. Jiuhong Chen for her valuable advice in revising the manuscript.

\section{References}

1. Schepkin VD, Ross BD, Chenevert TL, et al.: Sodium magnetic resonance imaging of chemotherapeutic response in a rat glioma[J]. Magn Reson Med. 2005, 53:85-92. 10.1002/mrm.20332

2. Moffat BA, Chenevert TL, Lawrence TS, et al.: Functional diffusion map: a noninvasive MRI biomarker for early stratification of clinical brain tumor response. Proc Natl Acad Sci U S A. 2005, 102:5524-9.

3. Rose AM, Valdes R: Understanding the sodium pump and its relevance to disease. Clin Chem. 1994, 40:16741685.

4. Boada FE, LaVerde G, Jungreis C, et al.: Loss of cell ion homeostasis and cell viability in the brain: what sodium MRI can tell us. Curr Top Dev Biol. 2005, 70:77-101. 10.1016/S0070-2153(05)70004-1

5. Thulborn KR1, Lu A, Atkinson IC, Damen F, Villano JL: Quantitative sodium MR imaging and sodium bioscales for the management of brain tumors. Neuroimaging Clin N Am. 2009, 19:615-624. 10.1016/j.nic.2009.09.001 


\section{Cureus}

6. Ouwerkerk R, Bleich KB, Gillen JS, Pomper MG, Bottomley PA: Tissue sodium concentration in human brain tumors as measured with 23Na MR imaging. Radiology. 2003, 227: 9-537. 10.1148/radiol.2272020483

7. Koike T, Tanaka S, Oda T, Ninomiya T: Sodium overload through voltage-dependent $\mathrm{Na}(+)$ channels induces necrosis and apoptosis of rat superior cervical ganglion cells in vitro. Brain Res. 2000, 51:345-355.

8. Madelin G, Regatte RR: Biomedical applications of sodium MRI in vivo . J Magn Reson Imaging. 2013, 38:511-529. 10.1002/jmri.24168 